\title{
Study of MANET Routing Protocols by GloMoSim Simulator
}

\author{
Ashwini K. Pandey \\ Hiroshi Fujinoki (*** correspondence author $* * *)$ \\ Department of Computer Science \\ Engineering Building, EB 2067 \\ Southern Illinois University Edwardsville \\ Edwardsville, Illinois 62026-1656
}

Phone: 618 650-3727

FAX: 618 650-2555

E-mail: \{apandey,hfujino\}@siue.edu

\begin{abstract}
This paper compares Ad hoc On-demand Distance Vector (AODV), Dynamic Source Routing (DSR), and Wireless Routing Protocol (WRP) for MANETs to Distance Vector protocol to better understand the major characteristics of the three routing protocols, using a parallel discrete event-driven simulator, GloMoSim. MANET (mobile ad hoc network) is a multi-hop wireless network without a fixed infrastructure. There has not been much work that compares the performance of the MANET routing protocols, especially to Distance Vector protocol, which is a general routing protocol developed for legacy wired networks. The results of our experiments brought us nine key findings. Followings are some of our key findings: (1) AODV is most sensitive to changes in traffic load in the messaging overhead for routing. The number of control packets generated by AODV became 36 times larger when the traffic load was increased. For Distance Vector, WRP and DSR, their increase was approximately 1.3 times, 1.1 times and 7.6 times, respectively. (2) Two advantages common in the three MANET routing protocols compared to classical Distance Vector protocol were identified to be scalability for node mobility in end-to-end delay and scalability for node density in messaging overhead. (3) WRP resulted in the shortest delay and highest packet delivery rate, implying that WRP will be the best for real-time applications in the four protocols compared. WRP demonstrated the best traffic-scalability; control overhead will not increase much when traffic load increases.
\end{abstract}

\section{Key Words}

Ad hoc networks, MANET, routing protocols, proactive routing protocols, reactive routing protocols 


\section{INTRODUCTION}

With the growing popularity and falling prices of the mobile hand-held computing and information exchange devices, the need and capability of these devices are also growing. These growth and need are creating its own set of new problems and challenges. Some examples of recent and not so recent wireless devices are cellular phones, personal digital assistants, tablet PCs, and lap-top PCs. All of these have the capability and need to transfer information over wireless medium to each other in a network. Currently, the wireless networks that allow communication between mobile devices can be classified into the following two categories:

(i) Networks having a fixed infrastructure: an example of such a network is a cellular phone network. A mobile cellular phone depends on a fixed infrastructure of base stations that cover fixed areas. A mobile phone communicates with the nearest base station and the base station in turn transmits the information to another base station, wired network, or to another mobile phone. When a mobile phone is at an intersection of the coverage areas of two base stations, it is switched to the base station with the stronger signal without any break in the communication and without the user becoming aware of it.

(ii) Networks that do not have a fixed infrastructure: this is an emerging but highly useful and promising type of network communication method. There are several situations where such a network would be indispensable; mostly, in unplanned events like natural disasters and wars, but

also in a planned event. For example, a meeting of businessmen scattered over a large place having no fixed infrastructure will be best supported by this kind of networks. This type of networks can be described as a network of mobile devices that is created or destroyed as needed and hence it is named Mobile Ad-hoc Network or MANET. 
The most distinguishing aspect of MANET is the lack of any fixed infrastructure and any central controlling authority. When there is no central controlling authority, the devices comprising a network are all equal and in such a situation, any decision needed to maintain a network becomes distributed. This creates the need for distributed routing algorithms, resource allocation schemes, network entry and exit rules, and network security. Moreover, as it is quite possible that a majority of the mobile devices in such a network will be hand-held devices, the need to conserve battery power will drive down the transmission power of the individual devices. Consequently, communication between two devices would often require relay by intermediate devices, which introduces the problem of multi-hop routing.

In wireless networks, physical links do not exist and a single transmission of a packet will transfer a packet to multiple nodes within the communication range of a transmitting node at the same time. We call this inherent broadcast of MANETs "local broadcast" to distinguish it from global broadcast. It is guaranteed that at least a copy of a packet will reach a destination node if every intermediate node, except the destination, repeats local broadcast without any explicit routing, as long as such a path exists. However, routing is still needed for MANETs because of the following reasons. If packets are transmitted by global broadcasts, excess copies of each packet will be transmitted in the network and to the destination. Thus, global broadcasts will entail unnecessary transmissions of packets, which waste battery power of intermediate nodes for transmitting duplicated copies of packets at the same time that wastes transmission bandwidth.

Several routing protocols have been proposed for the mobile ad hoc networks $[1,2,3,4]$. These can be categorized as the proactive (also known as the table driven) protocols, the reactive (known as source initiated or demand driven) protocols or the hybrid of the reactive and proactive protocols. A categorization of the prominent ad hoc routing protocols is shown in Figure 1. 
Proactive protocols: In proactive routing protocols, routing information to reach all the other nodes in a network is always maintained in the format of the routing table at every node. When the network topology changes (i.e., existing nodes have moved, some new links have been created or existing ones are dropped), such changes in link states are announced to all the nodes in a network. Thus, routes to all possible destinations are discovered in advance of packet transmissions.

If a proactive protocol is used for MANETs, an immediate problem is that rapid changes in network topology might overwhelm the network with control messages (messages for updating the routing table at every node) and the excess messaging overhead will compromise the throughput of actual data transmissions. Examples of proactive protocols are DV (Distance Vector) protocol [5], DSDV (Destination Sequenced Distance Vector) protocol [6], WRP (Wireless Routing Protocol) [7], and FSR (Fisheye State Routing) protocol [8]. The four protocols are also called table-driven protocols since the routing table will be updated for each change in link states in a network and routes are discovered using information stored in routing tables.

Reactive protocols: As its name suggests, this type of protocols discovers a route only when actual data transmission takes place. When a node wants to send information to another node in a network, a source node initiates a route-discovery process. Once a route is discovered, it is maintained in the temporary cache at a source node unless it is expired or unless some event happens (e.g., a link failure) that requires another route discovery to start over again. Reactive protocols require less amount of routing information at each node, compared to proactive protocols, as there is no need to obtain and maintain the routing information for all the nodes in a network. Another advantage in reactive protocols is that intermediate nodes do not have to make routing decisions.

An obvious disadvantage in reactive protocols is delay due to route discovery, called route acquisition delay. Furthermore, if routing information changes frequently, as it is the case in 
MANETs, and if route discoveries are needed for those changed routes, reactive protocols may result in a large volume of messaging overhead, since route recoveries require global broadcasts. Currently popular reactive protocols are DSR (Dynamic Source Routing) protocol [3], AODV (Ad hoc On Demand Distance Vector) protocol [2], and ABR (Associativity Based Routing) protocol [9].

Hybrid (combination of proactive and reactive) Protocols: Because of the initial delay due to route discovery and high control overhead in reactive protocols, a pure reactive protocol may not be the best solution for routing in MANETs. On the other hand, a pure proactive protocol used for a large network may not be feasible because of the need to keep a large routing table at all times. A protocol that uses the best features of both reactive and proactive protocol may be a better solution for MANETs. An example for such an approach is the ZRP (Zone Routing Protocol) [10], although it is not the panacea for all the limitations of other protocols.

Performance comparisons for ad hoc routing protocols have been reported in the recent past [11, $12,13,14,15,16]$. A comparison of DSR and AODV with some other protocols showed that the performance of DSR was superior to AODV when node mobility was high, although DSR had higher routing overhead as compared to AODV [11]. In a similar work by Das [12], it was observed that, for metrics like delay and throughput that have real life application implications, DSR performed better than AODV in conditions where the node density and/or node mobility were low. According to Das, DSR always generated less control messages for routing than AODV. However, Das argued that AODV resulted in less control messages than DSR under high traffic load and high node mobility.

We are not aware of any previously published work that measures how much better WRP, DSR and AODV protocols are than a classical Distance Vector protocol in ad-hoc networks. For example, how much better those MANET routing protocols will be than a classical Distance Vector 
protocol, in what aspects they are better than Distance Vector protocol and in what conditions those MANET routing protocols will be better than Distance Vector protocol, surprisingly have not been answered. In this paper, we try to find answers for such unanswered but significant questions to understand the advantages of the MANET routing protocols. In addition to those goals, we try to understand the major properties in the existing MANET routing protocols.

The rest of this paper is organized as follows. In section 2, the four routing protocols compared in this paper are described regarding their procedure and data structure to clarify the design motivations and characteristics in each of the four protocols. Section 3 describes our simulation experiments. In the section, experiment modeling and experiment designs are described followed by the key observations obtained from our experiments. Section 4 presents conclusions of this paper and a list of future work. Section 5 is a list of references.

\section{FOUR ROUTING PROTOCOLS COMPARED}

In this section, the four existing routing protocols we compared are described in terms of their implementation, design motivations and the major known characteristics to provide a foundation for comparisons.

Distance Vector (DV) protocol: Distance Vector protocol is a classic routing protocol whose refined versions are used in the current wired networks. It is a proactive protocol and is based on the concept of distance vector: every node in a network maintains a distance table (a onedimensional array or a vector, called distance vector), where each entry in a distance table contains the shortest distance and the address of the next-hop router on the shortest path to every destination in a network. 
In Distance Vector protocol, each node knows only the distance to its directly connected neighbors at the beginning. The distance vector initially contains only the distance to the direct neighbors (the distance to all other nodes is initialized to be infinity). Every node exchanges its distance vector with all its directly connected neighbors. After a node receives a distance vector from a neighbor node, the node updates its own distance vector to reflect the least cost path to other nodes that are not immediate neighbors. This process repeats until there is no more update in the distance vector at all nodes in a network. When this process is completed, each node will have a distance vector that contains the least cost path to all the other nodes in the network. When routing information changes at any node (for example, link failures), a node sends its new distance vector to all of its immediate neighbors. The new distance vector will be propagated to all the other nodes in a network using the same procedure to propagate the distance vector.

Distance Vector protocol has several advantages for MANET wireless networks. First of all, the protocol does not require a global broadcast, which is the property most essential for a routing protocol for large networks. Another advantage is the short route acquisition delay. Since this protocol is proactive, routing information for every destination should be available in the routing table at each node. The lack of need for route discovery on demand results in short route acquisition delay. The above two advantages also imply traffic load scalability, since the messaging overhead of the protocol will be constant irrespective of traffic load as long as there is no change in link states in a network.

The major known disadvantages in Distance Vector protocol are as follow. The convergence time for propagating routing information will be long especially when the link cost is increased [17]. Due to the long convergence time, it is possible that another change in link states occurs while the information for the previous change in link cost has not been completely propagated to 
the entire network. This could cause an erroneous routing decision, well known as "counting-toinfinity problem”, which can result in temporary routing loops.

Another disadvantage in Distance Vector protocol is the non-availability of alternative paths. Since the protocol uses a distributed approach, each node does not maintain the complete information about link states in a network. Lack of complete knowledge of the link states for all links in a network, each node is not aware of alternative paths to reach a destination. The unavailability of the information for multiple alternative paths to reach a destination (if they exist) will make the process of finding an alternative path during a sudden link failure a time-consuming process, if not impossible.

The third problem is the large routing table. For ad-hoc networks as MANET, the contents of the routing tables will be short-lived. Maintaining large routing tables, while their contents dynamically change in a short time, will result in high but unnecessary maintenance overhead. Finally, Distance Vector protocol assumes symmetric links (e.g., costs of links are same for the two directions on a link), which is not necessarily the case for wireless networks. This is because each transmitting host usually uses different signal frequency in wireless networks even when two hosts communicate with each other. Because of these problems, Distance Vector protocol is seldom used in its original form.

Wireless Routing Protocol (WRP): WRP is proposed by Murthy [7]. WRP is an extension of distance vector protocol that eliminates possibility of routing loops. Nodes in a network using WRP maintain a set of four tables:

(i) Link Cost Table: This table contains the cost of the link to each immediate neighbor node and information about the status of the link to each immediate neighbor.

(ii) Distance Table: The distance table of a node contains a list of all the possible destination nodes and their distances beyond the immediate neighbors. 
(iii) Routing Table: The routing table contains a list of paths to a destination via different neighbors. If a valid path exists between a source and a destination node, its distance is recorded in the routing table along with information about the next-hop node to reach the destination node.

(iv) Message Retransmission List (MRL): The MRL of a node contains information about acknowledgement (ACK) messages from its neighbors. If a neighbor doesn't reply with an ACK for a hello message within a certain time, then this information is kept in its MRL and an update is sent only to the non-responding neighbors.

WRP works by requiring each node to send an update message periodically. This update message could be new routing information or a simple "hello" if the routing information hasn't changed from the previous update. After sending an update message to its all neighbors, a node expects to receive an ACK from all of them. If an ACK message does not come back from a particular neighbor, the node will record the non-responding neighbor in MRL and will send another update to the neighbor node later.

The nodes receiving the update messages look at the new information in the update message and then update their own routing table and link cost table by finding the best path to a destination. This best path information is then relayed to all the other nodes so that they can update their routing tables. WRP avoids routing loops by checking the status of all the direct links of a node with its direct neighbors, each time a node updates any of its routing information.

Dynamic Source Routing (DSR) protocol [3]: Dynamic Source Routing protocol, as its name implies, is a source routing protocol: a complete sequence of intermediate nodes from a source to a destination will be determined at a source node and all packets transmitted by a source node to a destination follow the same path. Every packet header contains the complete sequence of nodes to reach a destination. DSR protocol is a reactive protocol and its primary motivations are, (1) to 
avoid periodic announcements of link states required in proactive protocols, by separating route discovery from route maintenance, (2) to avoid long convergence time of routing information and (3) to eliminate a large routing table for forwarding packets at intermediate nodes. The routing table, in a sense that it is the data structure to always hold routing information to reach every possible destination in a network, is not used in DSR protocol. In DSR, routes are discovered on demand and route cache is used to hold routes that are currently in use.

As most of the reactive protocols do, DSR consists of two procedures: route discovery and route maintenance.

Route Discovery: Every node in a network maintains a route cache that contains a list of hop-byhop routes to each destination node currently active and its expiration time (i.e., the time after which a route is considered stale and discarded). Before a source node starts transmitting data to a destination node, it first looks up its route cache to see if a valid route to that destination exists. If such a route exists, then the route discovery process ends and the source starts transmitting data using the route found in its route cache. Otherwise, a source node broadcasts a route request packet (RRP) to find a route to reach the destination. This broadcast is a global broadcast, which floods RRP in a network through all alternative paths to reach a destination. While a RRP is being broadcast and propagated to the destination, a RRP adds the address of every node it encounters to its list. If the same address appears more than once in the list, a RRP drops itself to avoid a routing loop. When a RRP reaches the destination node, the destination returns the learned path extracted from the RRP to the source node. For wireless networks that consist of asymmetric links, the destination node can send that path information back to the source node as a global broadcast, which allows DSR to work for asymmetric links.

Route Maintenance: Route maintenance in DSR is a mechanism to inform network failures to all nodes in a network. Its primary motivation is to expedite detection of network failures by 
explicitly announcing them to every node in a network using global broadcasts. No matter if it is a link or node failure, a node that is connected to the other end of a failed link is responsible for detecting a failure in DSR. On detecting a network failure, the detecting node broadcasts an error message, called error packet, to all the other nodes in a network to inform the failure. When other nodes receive an error packet, they will disable the paths that go through the failed link in their route cache.

An obvious advantage in DSR is that source nodes are aware of existence of alternative paths, which implies that recovery from a link failure will be easy and quick. Another advantage is that there will not be a chance of a routing loop (or it is easy to detect and avoid one). Furthermore, nodes do not have to maintain routing table, which is an advantage especially for a large network where nodes continue to move.

Being a reactive protocol also means that a route is stored in the route cache only when one is needed, which implies low maintenance overhead. Since most routes are short-lived and network topology frequently changes in MANETs, on-demand routing will make more sense than proactive protocols in terms of maintenance overhead for routing information at each node (this is because a node does not have to modify anything if a failed and/or changed link is not a part of any active path from this node).

The disadvantage in DSR is long route acquisition delay due to route discovery if short transmission delay is a significant factor. Long route acquisition delay may not be acceptable in certain situations, such as mobile communication at a battlefield. It is also quite possible that the path between a source and a destination may not be the shortest path (this is because resumed links will not be explicitly advertised in DSR), resulting paths with in suboptimal end-to-end delay. Another disadvantage is that messaging overhead of the protocol will be high during busy time, when many connections must be established in a short time since broadcast is used in route 
discovery. Large packet header will also cause low payload utilization, since each packet has to contain a list of all the intermediate routers to reach a destination.

Ad hoc On Demand Distance Vector Routing [2]: Ad-hoc On Demand Distance Vector (AODV) protocol is a reactive routing protocol that has a motivation of providing a good compromise between reactive source routing protocols and proactive protocols. The trade-off problem AODV addresses is the one between high messaging overhead due to periodic announcements of links states in proactive protocols and the large packet header to contain the entire route information to reach a destination in source routing protocols. Unlike pure distance vector protocols, routes are discovered and maintained on demand in AODV. Different from DSR, AODV uses a distributed approach, meaning that source nodes do not maintain a complete sequence of intermediate nodes to reach a destination. Different from Distance Vector and WRP, each path is established as a pair of two streams of pointers chained between a source and a destination node (details of this are described in a later section), which eliminates need of broadcasting error packets on a link failure. Similar to DSR, AODV uses the route discovery and route reply mechanism to create and maintain a route on demand.

Route Discovery: When a source node wants to send information to a destination node, it first looks up its own routing table to see if a valid route exists. If a valid route does not exist, a source node broadcasts a route request message that contains the source address, source sequence number, destination address, destination sequence number, broadcast ID, and hop count. The combination of the source address and the broadcast-ID is used to uniquely identify each route request message while a route request message is globally broadcast. Any node that has a valid route to the destination or the destination node is supposed to respond to route request messages by sending a route reply message. 
During a route discovery, two pointers are set up at every intermediate node between the source and the destination nodes. The two pointers are the back pointer and the forward pointer. A chain of the forward pointers is set up between a source and destination node while a route request message propagates from the source node to a destination. Similarly, a chain of the back pointers is set up while a route reply message propagates back from the destination (or from a node that already has a valid route to the destination) to the source. As a result, all the intermediate nodes on a route maintain a pair of the forward pointer and the back pointer for every connection that goes through them.

Every route request contains a list of intermediate nodes that have been encountered. If the same intermediate node appears more than once in the list, the route request message will be dropped (the chain of forward pointers must be deleted for a route request message to be deleted). This guarantees loop-free routing.

Route Maintenance: The route maintenance is performed using three different types of messages: route-error message, "hello" message and route time-out message. The purpose of the time-out message is obvious: if there is no activity on a route for a certain amount of time, the route pointers at the intermediate nodes will time out and the link will be deleted at the intermediate nodes. The periodic "hello" messages between immediate neighbors are required to prevent the forward and backward pointers from expiration. If one of the links in a route fails, a route-error message is generated by the node upstream (i.e., from an intermediate node to source nodes on the link and the message is propagated to every source node in its upstream that uses the failed link. Thus, the error packets will not be globally broadcast in AODV. Then, the source nodes in the upstream will initiate the route discovery process.

Primary advantages in AODV protocol are as follow. Route caches are small in AODV, because of its on-demand routing. Routes are guaranteed to be loop-free and valid. Convergence time is 
short for propagating changes in link states because link failure information will be propagated only to the nodes that are using a failed link (i.e., no broadcast for error packets). Information of a link failure will be propagated following the back pointers to reach such nodes. This implies that messaging overhead to announce link failures will be less than that of DSR, where link failure information is broadcast. As another advantage, each data packet does not contain the complete list of all the nodes on a route in AODV, which reduces the size of message packet. Similar to DSR, a source node is aware of multiple alternative paths.

One of the disadvantages in AODV protocol is that nodes can not perform routing (forwarding) packets as aggregate (at least in the latest existing implementation of AODV). This is because a set of pointers is used to maintain a route and each "flow" requires its own pair of back and forward pointers. For the nodes where a large number of connections exist, overhead for maintaining pairs of two pointers will be significant and may not be traffic-load scalable. Another disadvantage is longer route acquisition delay compared to that for proactive protocols since route discovery still must take place on demand. Different from DSR, AODV requires periodic "hello" messages to maintain pointers set up at every node on a path. Use of broadcast during route discovery, which contributes to high messaging overhead, is still the major overhead. Table 1 summarizes the discussions regarding the four routing protocols in this section.

\section{SIMULATION EXPERIMENTS}

To compare the performance of the four routing protocols described in the previous sections, simulation experiments were performed. In this section, experiment modeling, design and key observations from our simulation experiments are described in that order. 


\subsection{Experiment Modeling}

To compare the four routing protocols, a parallel discrete event-driven simulator, GloMoSim, was used. GloMoSim (Global Mobile Information System Simulator) is a simulation tool for large wireless and wired networks [18]. Our simulation experiments were executed on two desktop PCs: one running on a Pentium 3 processor, $850 \mathrm{MHz}$ with $512 \mathrm{MB}$ RAM and the other on a Pentium 4 processor, $3.2 \mathrm{GHz}$ with $2 \mathrm{~GB}$ RAM.

We focused on three performance measurements to compare the four routing protocols: mean end-to-end delay, packet delivery rate and routing overhead as measured by the number of control packets generated for routing. The three measurements in our experiments were defined as follows:

(i) End-to-end Delay: The average time from the beginning of a packet transmission (including route acquisition delay) at a source node until packet delivery to a destination.

(ii) Packet Delivery Rate: Packet delivery rate is the ratio of the number of user packets successfully delivered to a destination to the total number of user packets transmitted by source nodes.

(iii) Messaging Overhead: The number of control packets generated for routing by each routing protocol.

The control parameters we used in our simulation experiments were traffic load, node density and node mobility. Then, mean end-to-end delay, packet delivery rate and routing overhead were measured for various node mobility (Experiment \#1) and node density (Experiment \#2) for three different levels of traffic load.

Traffic load generated by each source node was modeled by a constant bit rate data stream, whose transmission rate was defined by packet transmission interval for fixed size packets. Three 
different levels of traffic load defined by the packet transmission intervals are, (i) low traffic load: one packet transmitted at every 10 seconds, (ii) medium traffic load: one packet at every second, and (iii) high traffic load: one packet at every 0.1 second.

Movement of each node was modeled using the random waypoint model [3]. In the random waypoint model, each node remains stationary for the duration of its "pause-time". At the end of a pause time, a node starts moving in a randomly selected direction in the network terrain at a fixed speed. Once a node reaches its new location, it remains stationary during its next pause-time. At the end of the new pause time, a node again starts moving in another randomly selected direction in the network. This movement process was continued during a simulation experiment.

The network terrain size was fixed for $2,000 \times 2,000$ meters. The radio signal transmission range was fixed at 175 meters (radius of 175 meters). The transmission bandwidth of each link was fixed at $2 \operatorname{Mbps}\left(=2 \times 10^{6}\right.$ bits per second) and the simulation time was 500 seconds for all the experiments. In every experiment, there were 25 randomly selected pairs of a sender and a receiver node. Traffic load was simulated in such a way that each of the 25 sources transmitted a constant bit rate data stream to one of the randomly selected 25 receivers at approximately the same time. Data packet size was fixed at 1,460 bytes. The above parameters were used for all of our simulation experiments.

To avoid the experimental artifact of having a network that was totally devoid of any traffic before a simulation experiment began, a random pattern of FTP and HTTP network transmission was simulated for 10 seconds. This involved sending data packets of size 1,460 bytes from randomly selected nodes to randomly selected destinations for random duration using either FTP or HTTP. This simulated a real network that was already in use before a simulation experiment began.

In our experiments, TCP was not used as the transport-level protocol, because the main objective in this project was to observe the net characteristics of each routing protocol. Since TCP performs 
flow control and packet re-transmission, it would have prevented us from observing essential characteristics (such as the number of packets actually transmitted and dropped) of the four routing protocols. To directly observe the major characteristics of the routing protocols, we selected UDP for the transport layer in our experiments. IEEE 802.11 was used for the MAC layer protocol. For radio layer protocol, "no capture" and "free space" mode was used for the propagation mode.

\subsection{Experiment Design}

In our simulation experiments, the following two experiments, mobility tests (Experiments \#1) and node density tests (Experiments \#2), were developed. The two experiments were defined in the following sections:

Experiment \#1 (Mobility Tests): Effect of node mobility was studied. In this experiment, there were 50 nodes moving using the random waypoint model described before. The speed of node movements was $45 \mathrm{~km} / \mathrm{h}$, with the following five levels of node mobility: (i) perpetual mobility: move pause time was 0 second, (ii) high mobility: move pause time was 120 seconds, (iii) medium mobility: move pause time was 300 seconds, (iv) low mobility: move pause time was 400 seconds and (v) zero mobility: move pause time was 500 seconds.

Performance of the four routing protocols was compared for end-to-end delay, packet delivery rate and messaging overhead for various combinations of different levels of traffic load and node mobility.

In Experiment $\# 1$, ten experiment runs were repeated for each experiment configuration, with a different starting random seed value for each experiment run. Different initial seed values ensured that network started out with different node placements and different starting directions for their 
movement. The obtained results were averaged. The most extreme results (highest and lowest values) were discarded.

For the table driven protocols (Distance Vector and WRP), it was expected that messaging overhead would not change significantly when the traffic load was increased. This is because the number of routing updates for these protocols is dependent only on changes in link states in a network, which is independent of traffic load. Since WRP uses periodic control packets to maintain routes, it was expected that WRP would have higher messaging overhead than Distance Vector. For AODV, we expected that messaging overhead would increase with increased traffic load as AODV uses periodic messages to check the consistency of the network, which would increase when the network traffic load increased. For DSR, since there is no periodic route maintenance required, it was expected that the control overhead in DSR would be less than that of AODV.

Experiment \#2 (Node Density Tests): In this experiment, the effects of node density on mean end-to-end delay, the packet delivery rate and routing overhead were measured for the four routing protocols. Effect of node density was studied while all the nodes in a network were moving at a velocity of $45 \mathrm{~km} / \mathrm{h}$ with no pause (i.e., perpetual node mobility). The following three levels of node density were defined: (i) low-density networks: 50 nodes in a network, (ii) medium-density networks: 75 nodes in a network, and (iii) high-density networks: 100 nodes in a network. Similar to Experiment \#1, ten experiment runs were repeated for each experiment configuration. The same randomization technique and statistics methods as Experiment $\# 1$ were used. Simulations were performed with 100,75 , and 50 nodes while 25 nodes were acting as senders with another 25 randomly selected receiver nodes.

For the two reactive routing protocols (DSR and AODV) where the minimum-path routing is not guaranteed due to lack of explicit announcements of newly added nodes and links, increase in node 
density was expected to result in longer end-to-end delay than the proactive protocols. This is because the network diameter will be increased if number of nodes in a network is increased without increase in the number of links. Increase in the network diameter implies increase in the path lengths. Thus, we expected that the end-to-end delay in the two reactive protocols would be more affected by increase in the node density than the proactive protocols.

Another possible impact from the increase in the number of nodes is that messaging overhead for proactive protocols will increase since the table size will increase to account for extra nodes. For reactive protocols, increase in the number of nodes in a network will also increase the messaging overhead in global broadcast during route discovery, while this is not the case for proactive protocols. The node density tests tried to measure the impact of node density to proactive and reactive protocols.

\subsection{Experiment Results and Analysis}

Experiment \#1 (Mobility Tests): The left half of Table 2 (the columns under the index of "Node Mobility") shows the number of the control packets observed for the five different levels of node mobility. The left-most column, "Load", indicates three traffic load levels. Figure 2 shows the number of the control packets when the traffic load was low. The graphs show the minimum, the average, and the maximum values (after discarding the extremes) with error bars indicating approximate percent difference.

Table 2 shows that DSR resulted in the smallest number of control packets for all the experiments in the mobility tests. Under low and medium traffic load, WRP generated the largest number of control packets (1,276 and 1,285 packets for low and medium traffic load, when the node mobility was zero). Under the high traffic load, AODV generated the largest number of 
control packets $(3,899$ packets) followed by WRP $(1,246$ packets). One of the possible causes for high messaging overhead in WRP for low and medium traffic load is that WRP generated control packets to prevent loops in routing, which is not the case for the other three protocols.

It was observed that the impact of traffic load to routing overhead was more significant for AODV and DSR than for WRP and Distance Vector protocols. For AODV, the number of control packets steeply increased from 281 to 10,123 packets (which was approximately 36 times increase) when traffic load was increased from low to high at perpetual mobility (Table 2). The increase was from 108 to 822 packets for DSR (approximately 7.6 times increase). For Distance Vector and WRP, the difference was minor. For Distance Vector, it was decreased from 1,577 to $1,568(0.6 \%$ decrease). In WRP, it was decreased from 3,159 to 2,875 (9\% decrease). These results imply that WRP and Distance Vector are more scalable to increase in traffic load in terms of messaging overhead than AODV and SDR.

To study the effect of node mobility to messaging overhead, the ratio of the control packets generated at the perpetual mobility to that of zero mobility was calculated for each of the four protocols. The ratio of increase in the number of control packets in DSR was $207.7 \%(=108$ packets/52 packets), $128.8 \%(=452 / 351)$, and $157.5 \%(822 / 522)$ for low, medium and high traffic load (Table 2), when the node mobility was increased from zero mobility to perpetual mobility. For the same experiment, Distance Vector resulted in 252.6, 239.8, and 196.2\% increase, while WRP resulted in 247.7, 242.2, and 230.7\% and AODV in 180.7, 494.5, and 259.7\% increase. DSR demonstrated the lowest increase rate in the number of control packets when mobility was increased from zero to perpetual for medium and high traffic load $(209.7,128.8$ and $157.3 \%$ increase). This implies that DSR is least vulnerable to node mobility in terms of routing overhead in the four protocols. The increase rate of the control packets for WRP was least affected by the 
traffic load (only 17\% difference between the low and high traffic load), which implies that WRP will be traffic-load scalable.

The left half of Table 3 shows the packet delivery rate in percent for the four protocols for the five different levels of node mobility. Figure 3 shows the packet delivery rate of the four protocols observed for the low traffic load. Note that the packet delivery rate may not reach $100 \%$ even when any node in a network is not moving. In UDP, the packets dropped due to buffer overflow are not retransmitted, resulting in undelivered packets.

From Table 3, it can be seen that the packet delivery rate was low (between 12.0 to $17.1 \%$ ) for the four protocols when the node mobility was perpetual even at low traffic load. When the node mobility was zero and the traffic load was high, WRP resulted in higher packet delivery rate than Distance Vector protocol by $4.7 \%$ (19.1\% for WRP while it was $14.4 \%$ for Distance Vector), presumably due to WRP's better route convergence and maintenance than those used in Distance Vector protocol. AODV and DSR demonstrated similar results in the packet delivery rate, when traffic load level was low. The packet delivery rate for AODV was 13.8, 30.3, and 57.9\%, while it was $12.0,28.8$, and $60.3 \%$ for DSR for perpetual, medium and zero node mobility.

A possible reason for the reduced packet delivery rate in the two reactive protocols (i.e., AODV and DSR) was route fluctuation due to the delay needed for route recoveries. Paths detected by route discovery might change again before source nodes transmit packets if nodes were actively moving in a network. Detected changes in link states will be notified to source nodes, which will trigger the source nodes to initiate another route discovery. Thus, data packets that are transmitted before rediscovery of routes would be lost. For the two proactive protocols (i.e., DV and WRP), convergence delay in propagating updated routing information can be the primary cause of reduced packet delivery rate when node mobility was increased. Data packets transmitted while a latest 
routing information is being propagated in a network will be lost due to possible routing loops caused by temporary inconsistency in the routing tables.

Figure 4 shows the ratio of packet delivery rate observed at zero mobility to that of perpetual mobility in percent. We observed that the packet delivery rate of AODV was least affected by changes in traffic load. For AODV, the ratio was $419.6 \%, 365.3 \%$, and $481.5 \%$ for low, medium and high traffic load (for low traffic load, the ratio was calculated as 57.9 (the delivery rate of AODV at zero mobility)/13.8 (the delivery rate of AODV at perpetual mobility), which is 4.1956). The absolute difference between the maximum and minimum in percentage was 116.2 (481.5365.3). The increase was 706.5, 475.3, and 138.5\% for Distance Vector (the difference was 568.0), while it was 502.5, 319.0, and 291.9\% for DSR (the difference was 210.6) and 521.1, 333.0, and 214.6\% for WRP (the difference was 306.5). These results suggest that AODV will be one of the protocols whose packet delivery rate is least vulnerable to increase in traffic load.

The left half of Table 4 shows the mean end-to-end delay (in seconds) observed for the four protocols for the five different levels of node mobility. DSR resulted in the largest end-to-end delay while WRP the shortest. DSR resulted in the longest end-to-end delay except when traffic load was high at perpetual node mobility (for this case only, Distance Vector resulted in the longest delay). The delay from Distance Vector was 32.42 seconds, which was approximately two times longer than DSR. DSR resulted in 2.79, 2.10, and 1.52 seconds for perpetual, medium and zero mobility when traffic load was low. It was $0.28,0.15$, and 0.12 seconds for Distance Vector, while it was $0.15,0.10$, and 0.10 seconds for WRP and $0.56,0.53$, and 0.43 seconds for AODV for the same experiments. For medium traffic load, similar results were observed.

The end-to-end delay of WRP was significantly short compared to the other three protocols at the perpetual node mobility when the traffic load was high. The mean end-to-end delay of WRP was 
3.83 seconds, which was only $11.8,21.2$, and $24.7 \%$ of Distance Vector (32.42 seconds), AODV (18.04 seconds), and DSR (15.50 seconds) protocols, respectively.

Possible explanations for the short end-to-end delay in WRP are: (1) WRP is a proactive protocol, (2) WRP uses an efficient route maintenance method (no loops and constant check of link status), hence the average end-to-end delay would be less as the likelihood of sending information on invalid paths would be less, and (3) in case of link failures, WRP recovers much quickly [7]. These advantages are not present in Distance Vector protocol except for (1); hence Distance Vector resulted in the longer end-to-end delay than WRP especially at high traffic load and high mobility.

We also observed that Distance Vector constantly resulted in the highest increase rate in end-toend delay when node mobility was increased from zero to perpetual. Especially when the traffic load was high, Distance Vector showed the worst scalability for node mobility. In Distance Vector, the end-to-end delay increased at the highest rate when node mobility was increased from zero mobility to perpetual mobility. In other words, the other three routing protocols demonstrated that they had better scalability for node mobility in end-to-end delay, compared to Distance Vector. As a result, it is concluded that one of the advantages in the three exiting routing protocols for routing MANETs is their good scalability for node mobility in end-to-end delay.

Experiment 2 (Node Density Tests): The right half of Table 2 (the columns under the index of "Node Density") shows the number of control packets observed for the three different levels of node density. Figure 5 shows the number of control packets for the three levels of node density when traffic load was high. Figure 6 shows the ratio of the control packets generated at high node density to that generated at low density.

At low and medium traffic load, the table based protocols (Distance Vector and WRP) generated more control packets than the demand based protocols (AODV and DSR). For the low traffic load, Distance Vector generated 4,456, 2,598, and 1,438 packets for low, medium and high node density, 
while they were 2,512, 2,073, and 1,800 packets for WRP. AODV generated 307, 303, and 262 packets, while DSR resulted in only 95, 46, and 48 packets. Similar results were observed for medium traffic load.

The messaging overhead in AODV sharply increased when traffic load was increased. The number of control packets increased from 307 at low traffic load to 11,768 packets at high traffic load (at high node density for both), which was 38.3 times increase from low traffic load. Similar increase was observed for medium and low node density. These results imply that AODV is not traffic load scalable in terms of control overhead.

The ratio of control messages generated at the high density to that of the low density was calculated to measure the scalability in messaging overhead for node mobility. We defined good scalability in messaging overhead for node density to mean that number of control packets would not rapidly increase when node density was increased. Distance Vector resulted in the worst node density scalability, while DSR resulted in the best. When the node density was increased from low to high, Distance Vector resulted in $309.9 \%$ (4,456 divided by 1,438$), 353.6 \%$, and $328.4 \%$ increase in the number of control packets for low, medium and high traffic load. DSR resulted in increase only by 197.9, 151.9, and 210.7\%. AODV and WRP resulted in similar results. For AODV, the increase was $117.2,107.4$, and $124.8 \%$, while it was $139.6,123.6$, and $116.2 \%$ for WRP. The same pattern was observed for the three different levels of traffic load. These results suggest that an advantage in using the three routing protocols designed for MANETs (compared to a classic Distance Vector) is in the good scalability in control overhead for increased node density.

The right half of Table 3 shows the observed packet delivery rate for the four protocols for the three different levels of node density. The packet delivery rate increased as the node density increased for all the four protocols at any traffic load level. When both of the node density and traffic load were low, the packet delivery rate was $16.3,22.4,19.1$, and $16.2 \%$ for Distance Vector, 
WRP, AODV and DSR, respectively, while they were $24.2,26.3,21.8$, and $20.0 \%$ when the node density was increased to high. Similar results were observed for medium and high traffic load. For the effect of node density to packet delivery rate, we did not observe any significant difference in the four protocols.

The right half of Table 4 shows the mean end-to-end delay observed in Experiment \#2. For low traffic load, the increase in the end-to-end delay was minor for changes in node density for all the four protocols. The mean end-to-end delay for Distance Vector was $0.12,0.19$, and 0.17 seconds for high, medium and low node density, when traffic load was low. It was $0.11,0.15$, and 0.13 seconds for WRP. For AODV, it was 0.40, 0.36, and 0.41 seconds, while it was $1.72,1.54$, and 1.40 seconds for DSR. These results imply that the node density will not significantly affect endto-end delay if traffic load is low. However, the long end-to-end delay for DSR is conspicuous at low traffic load.

At medium traffic load, the higher node density resulted in lower end-to-end delay. For example, mean end-to-end delay for Distance Vector was $0.12,0.18$, and 0.14 seconds for high, medium and low density, while it was $0.28,0.49$, and 0.44 seconds in DSR (similar results were observed for WRP and AODV for the same experiments). One of the possible explanations for this result is the presence of a larger number of alternative paths to a destination node due to the increase in the number of nodes in a network.

At high traffic load, the end-to-end delay significantly improved (i.e., became shorter) as the node density increased except for AODV. Mean end-to-end delay for Distance Vector was 2.58, 4.60, and 5.17 seconds for high, medium and low node density. It was $3.12,5.59$, and 3.40 seconds for WRP. DSR resulted in 7.02, 10.83, and 7.48 seconds.

The ratio of the mean end-to-end delay observed at low node density to that of high node density for Distance Vector was 141.7, 116.7, and 200.4\% increase for low, medium and high traffic load. 
The ratio was $102.5,88.0$, and $72.8 \%$ for AODV. It was $81.4,157.1$, and $106.6 \%$ for DSR, and 113.3, 127.3, and 109.0\% for WRP. Especially for low and medium traffic load, the increase rate in end-to-end delay was not significant (less than 20\% increase) except for Distance Vector and DSR protocol at low and medium traffic load (141.7\% for Distance Vector at low traffic load and $157.1 \%$ for DSR at medium traffic load). Distance Vector resulted in a high increase rate in endto-end delay when traffic load was high.

\section{CONCLUSIONS AND FUTURE WORK}

This paper attempts to compare three representative routing protocols for MANETs (WRP, AODV and DSR) to Distance-Vector protocol in their packet delivery rate, end-to-end delay and messaging overhead to understand the advantages in the three protocols developed for MANETs. We developed two sets of experiments in this project. The first experiment compared the four routing protocols with respect to node mobility in an ad hoc network for different levels of traffic load. In the second experiment, the four protocols were compared for different node density for different levels of traffic load. The following is a list of key findings obtained from our experiments.

Finding \#1: Although DSR and AODV are both reactive protocols, DSR resulted in the best (i.e., the least) messaging overhead for all the experiments in both Experiment \#1 and \#2, and AODV generated higher volume of control packets even than the two proactive protocols (Distance Vector and WRP). Since the major difference between DSR and AODV for control overhead is the lack of periodic route maintenance in DSR, the periodic "hello" messages used in AODV to maintain routes was most probably responsible for DSR's high control overhead. 
Finding \#2: Contrary to our prediction, Distance Vector performed much better than expected. Distance Vector resulted in nearly the similar results in packet delivery rate (Figure 3) and even better in end-to-end delay, especially compared to the two reactive protocols (AODV and DSR). The category where Distance Vector mostly resulted in poor performance than the other three MANET protocols was the messaging overhead (in the number of control packets). DSR, against our expectation, resulted in worse performance in messaging overhead when traffic load was high. End-to-end delay for DSR was constantly longer than those of the three other protocols.

Finding \#3: The impact of traffic load to the amount of messaging overhead for routing was observed high in AODV. For AODV, the number of control packets increased to 36 times larger when the traffic load was increased from low to high in our experiments (Table 2). For Distance Vector, WRP and DSR, their increase was approximately 1.3 times, 1.1 times and 7.6 times, respectively. The experiment results show that proactive protocols (Distance Vector and WRP) were less vulnerable to increase in traffic load than reactive protocols.

Finding \#4: Experiment results suggest that DSR has the best scalability for node mobility in messaging overhead, meaning that the number of control packets in DSR will not increase sharply even when node mobility increases.

Finding \#5: The packet delivery rate was quite low (between 12.2 to $17.1 \%$ ) for all the four routing protocols when the node mobility was perpetual (i.e., when nodes continued to move). Since the packet delivery rate was low when traffic load was low and node mobility was perpetual, this low packet delivery rate was most likely because routing information was easily outdated and might not be updated quickly enough during extremely high node mobility. Thus, data packets were transmitted to non-existing paths, causing a high rate of lost packets.

Finding \#6: We found that the DSR had the longest end-to-end delay and that WRP had the shortest end-to-end delay for all the traffic loads. The end-to-end delay of WRP was significantly 
short compared to the other three protocols, when the traffic load was high at the perpetual node mobility. Possible explanations for the short end-to-end delay in WRP are: WRP maintains the best-path information to a destination, avoids routing loops during route discovery process, and converges quickly after a link failure.

Finding \#7: Results shown in Figure 6 suggest that one of the advantages in using the three routing protocols designed for MANETs is the good scalability for node density in messaging overhead. The results show that increase in the number of control packets was minor for the three protocols designed for MANETs (AODV, DSR and MRP) when node density was increased. The largest increase in the number of control packets when node density was increased from low to high was $124.8 \%$ for AODV, $210.7 \%$ for DSR and $139.6 \%$ for WRP, while it was $353.6 \%$ for Distance Vector.

Finding \#8: We observed that WRP resulted in a good packet delivery rate (WRP resulted in the best packet delivery rate expect when traffic load and node density were both medium). This result suggests that WRP will be a good protocol if the high reliability and throughput are the priority.

Finding \#9: Distance Vector resulted in the worst scalability for node mobility in the end-to-end delay. When the node mobility was increased from zero mobility to perpetual mobility, Distance Vector resulted in the highest increase rate in end-to-end delay. This inversely implies that one of the primary advantages in the three routing protocols designed for MANETs is the scalability for node mobility in end-to-end delay.

Future study includes measuring the actual number of bytes transmitted for control messages, which would be useful to better differentiate the two on-demand protocols. Another future work is to perform the experiments for various different node migration speeds. We used the node mobility of $45 \mathrm{~km} / \mathrm{h}$ in our experiments this time. However, the node migration speed of $45 \mathrm{~km} / \mathrm{h}$ is just one of the possible velocities. Keeping the migration speed lower may lessen or rule out the cases of 
packets getting dropped even before routing information is updated. This may affect the simulation results and perhaps will bring out the strengths and weaknesses of different protocols unambiguously.

\section{REFERENCES}

1. Perkins C. and Bhagwat P. Highly Dynamic Destination Sequenced Distance Vector Routing for Mobile Computers. Proceedings of the SIGCOMM '94 Conference on Communication Architectures, Protocols and Applications 1994; 234-244.

2. Perkins C. and Royer EM. Ad hoc On Demand Distance Vector (AODV) Routing. Proceedings of the Second IEEE Workshop on Mobile Computing Systems and Applications 1999; 90-100.

3. Johnson DB. and Maltz DA. Dynamic Source Routing in Ad-Hoc Wireless Networking, Mobile Computing; Kluwer Academic Publishing: New York, 1996.

4. Corson MS. and Ephremides A. A Distributed Routing Algorithm for Mobile Wireless Networks. ACM Baltzer Wireless Networks Journal 1995; 1(1):61-81.

5. Ford LR. and Fulkerson DR. Flows in Network; Princeton University Press: Princeton, NJ, 1962.

6. Murthy S. and Garcia-Luna-Aceves JJ. Loop-free Internet Routing Using Hierarchical Routing Trees. Proceedings of the IEEE INFOCOM 1997; 101-108.

7. Murthy S. and Gracia-Luna-Aceves JJ. An Efficient Routing Protocol for Wireless Networks. ACM Mobile Networks and Applications Journal, Special Issue on Routing in Mobile Communications Networks 1996; 183-197.

8. Iwata A., Chiang CC., Pei G., Gerla M., and Chen TW. Scalable Routing Strategies for Ad hoc Wireless Networks. IEEE Journal on Selected Areas in Communications, Special Issue on Wireless Ad hoc Networks 1999; 17(8):1369-1379.

9. Toh CK. A Novel Distributed Routing Protocol to Support Ad hoc Mobile Computing. Proceedings of the 1996 IEEE Fifteenth Annual International Phoenix Conference on Computers and Communications 1997; 1-36.

10. Peralman MR. and Haas ZJ. Determining the Optimal Configuration for the Zone Routing Protocol. IEEE Journal on Selected Areas in Communications, Special Issue on Wireless Ad hoc Networks 1999; 17(8):1395-1414.

11. Broch J., Maltz DA, Johnson DB., Hu Y., and Jetcheva J. A Performance Comparison of 
Multi-Hop Wireless Network Routing Protocols. Proceedings of the Fourth Annual ACM/IEEE international Conference on Mobile Computing and Networking 1998; 25-30.

12. Das SR., Perkins CE., and Royer EM. Performance Comparison of Two On-Demand Routing Protocols for Ad Hoc Networks. Proceedings of the IEEE INFOCOM 2000; 3-12.

13. Raju J. and Garcia-Luna-Aceves JJ. A Comparison of On Demand and Table Driven Routing for Ad Hoc Wireless Networks. Proceedings of IEEE International Conference on Communications 2000; 1702-1706.

14. Royer E. and Toh CK. A Review of Current Routing Protocols for Ad Hoc Mobile Wireless Networks. IEEE Personal Communications 1999; 46-55.

15. Hong X., Xu K., and Gerla M. Scalable Routing Protocols for Mobile Ad Hoc Networks. IEEE Network 2002; 11-21.

16. Cordeiro CM. and Agrawal DP. Mobile Ad Hoc Networking. Proceedings of the 20th Brazilian Symposium on Computer Networks 2002; 125-186.

17. Jaffe JM. And Moss FM. A Responsive Routing Algorithm for Computer Networks. IEEE Transaction of Communications 1982; 30(7): 1758-1762.

18. Zeng X., Bagrodia R., and Geria M. Glomosim: A Library for Parallel Simulation of Large Scale Wireless Networks. Proceedings of the 12th Workshop on Parallel and Distributed Simulations 1998; 154-161. 


\section{Figure and Tables}

- Figure 1 - Categorization of ad-hoc routing protocols

- $\quad$ Figure 2 - Control overhead for various node mobility levels at low, medium and high traffic load

- $\quad$ Figure 3 - Packet delivery rate for five different levels of node mobility at low traffic load

- Figure 4 - The relative increase in packet delivery rate when mobility was decreased to zero mobility

- $\quad$ Figure 5 - Messaging overhead for three different levels of node density at high traffic load

- Figure 6 - The ratio of the number of control packets observed at high node density to that observed at low node density

- Table 1 - Major properties of the four protocols compared

- Table 2 - Number of control packets observed for the five different levels of node mobility and four different levels of node density

- Table 3 - Packet delivery rate (in percent) for the five different levels of node mobility and three levels of node density

- Table 4 - Mean end-to-end delay (in seconds) for the five different levels of node mobility and node density 
Author Name: Fujinoki, Hiroshi

E-mail: hfujino@siue.edu

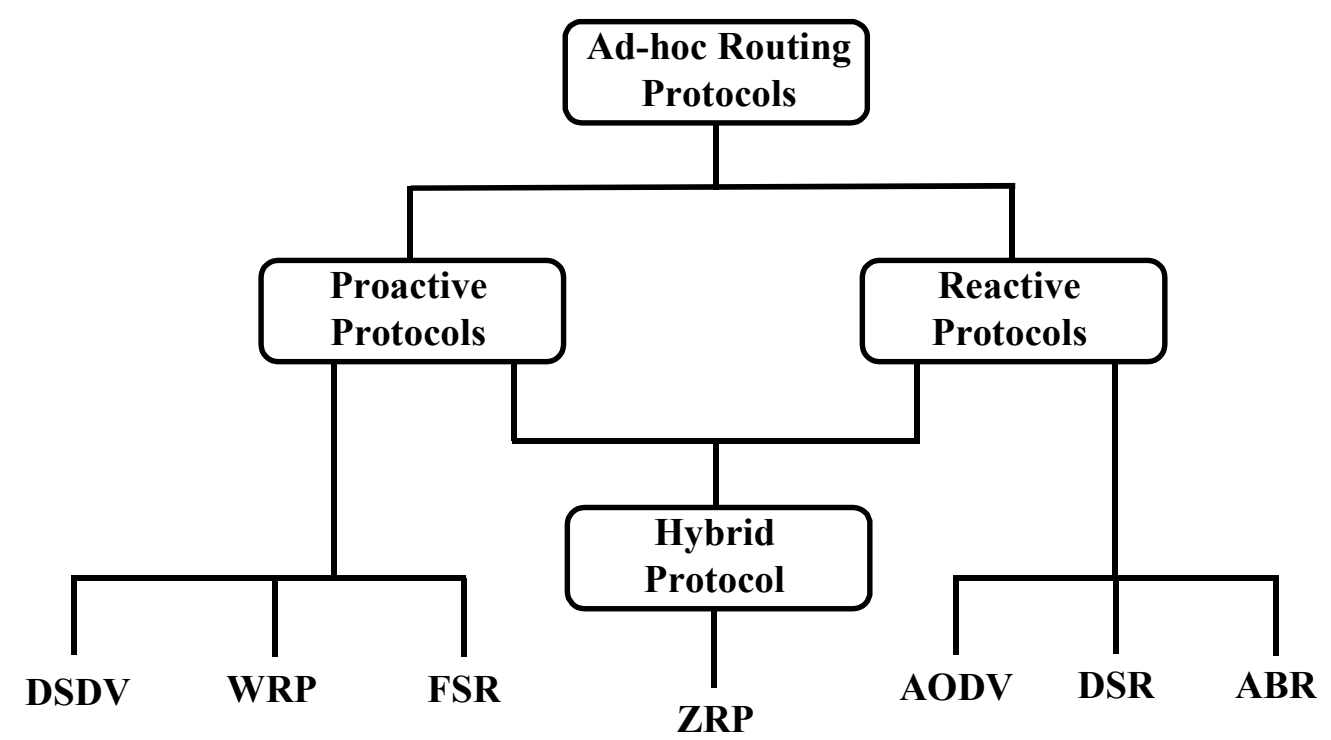

DSDV: Destination Sequenced Distance Vector WRP: Wireless Routing Protocol FSR: Fisheye State Routing AODV: Ad hoc On Demand Distance Vector DSR: Dynamic Source Routing ABR: Associativity Based Routing ZRP: Zone Routing Protocol

Figure 1 - Categorization of ad-hoc routing protocols 
Author Name: Fujinoki, Hiroshi

E-mail: hfujino@siue.edu

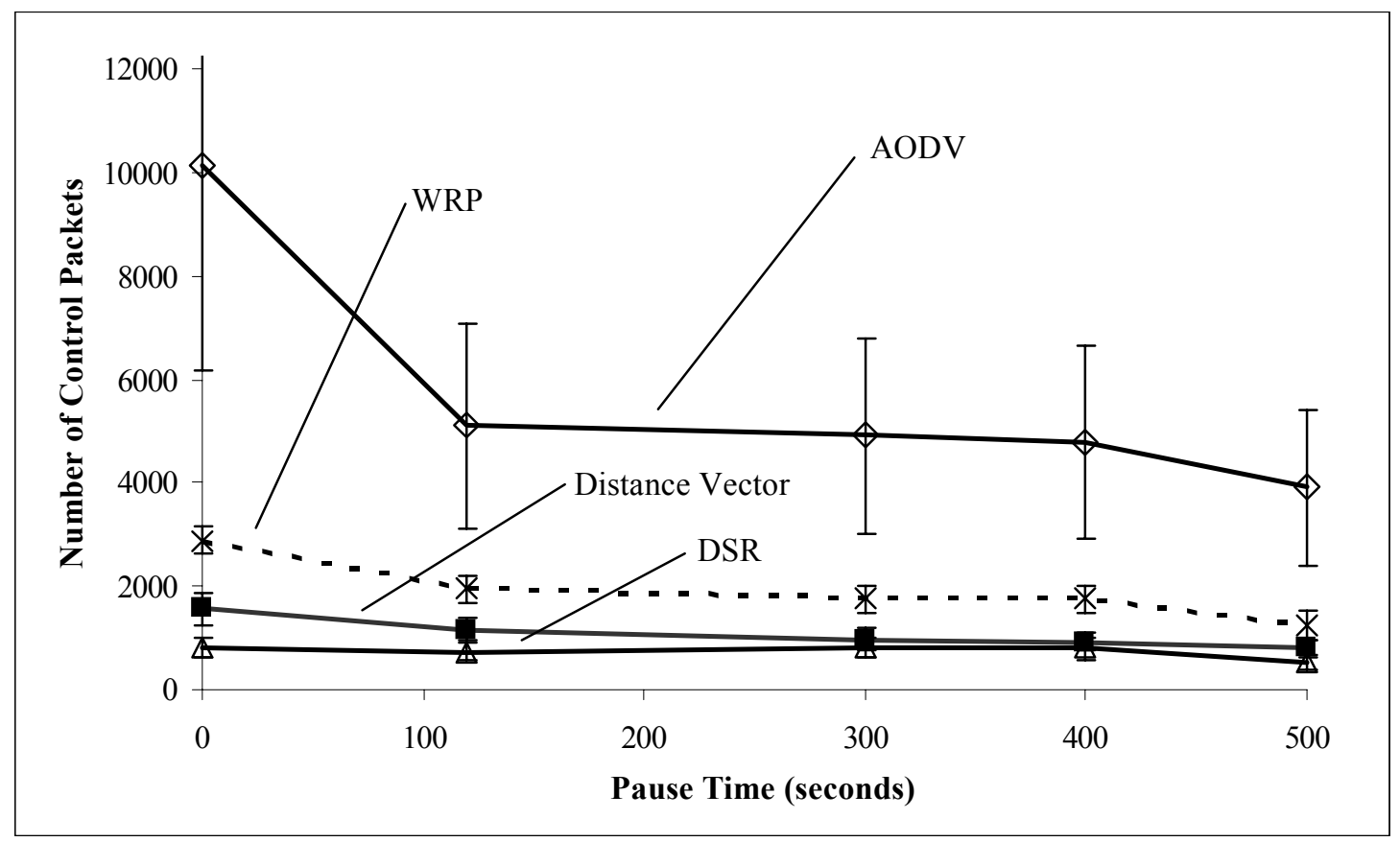

Figure 2 - Control overhead for various node mobility levels at three levels of traffic load 
Author Name: Fujinoki, Hiroshi

E-mail: hfujino@siue.edu

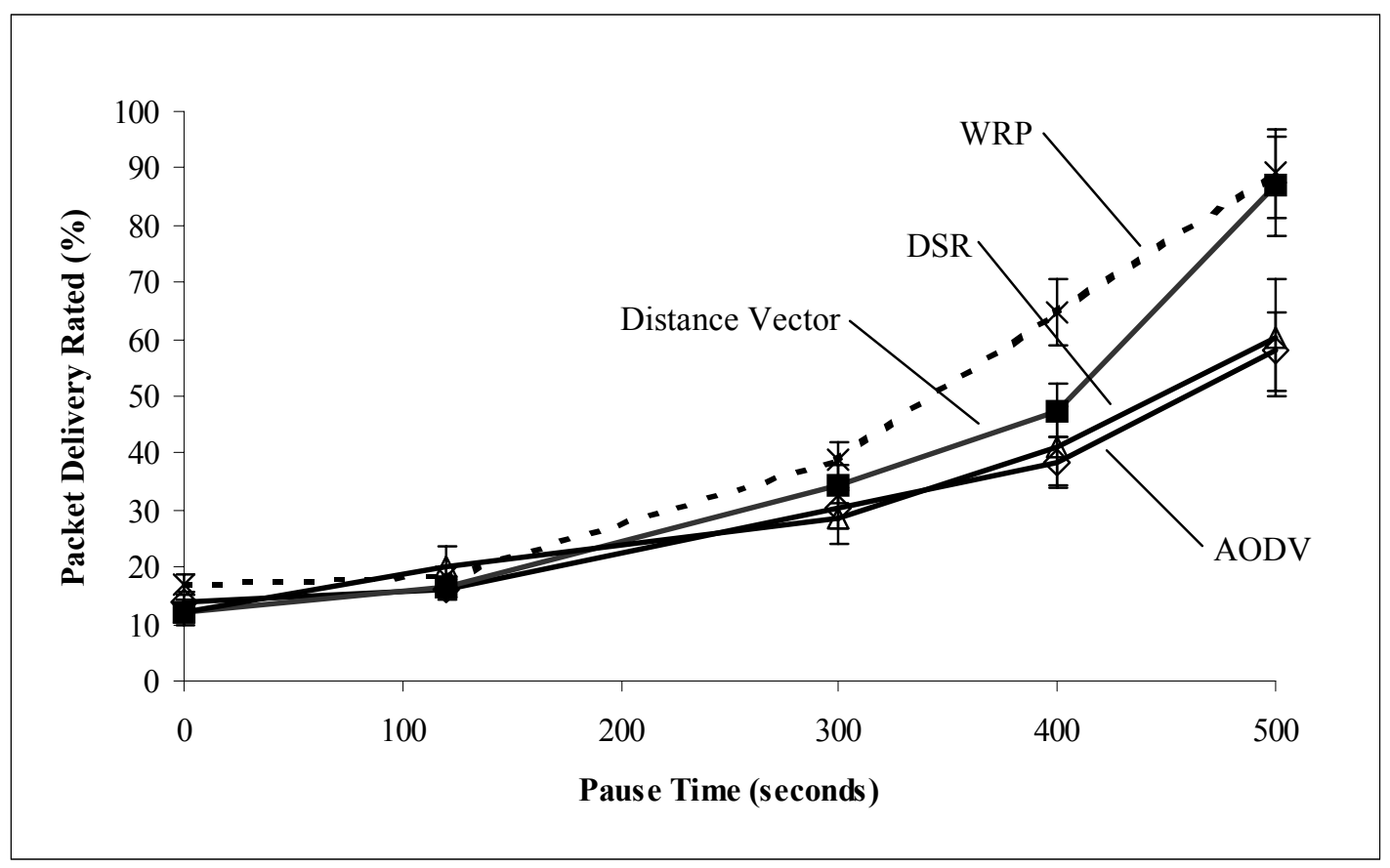

Figure 3 - Packet delivery rate for five different levels of node mobility at low traffic load 
Author Name: Fujinoki, Hiroshi

E-mail: hfujino@siue.edu

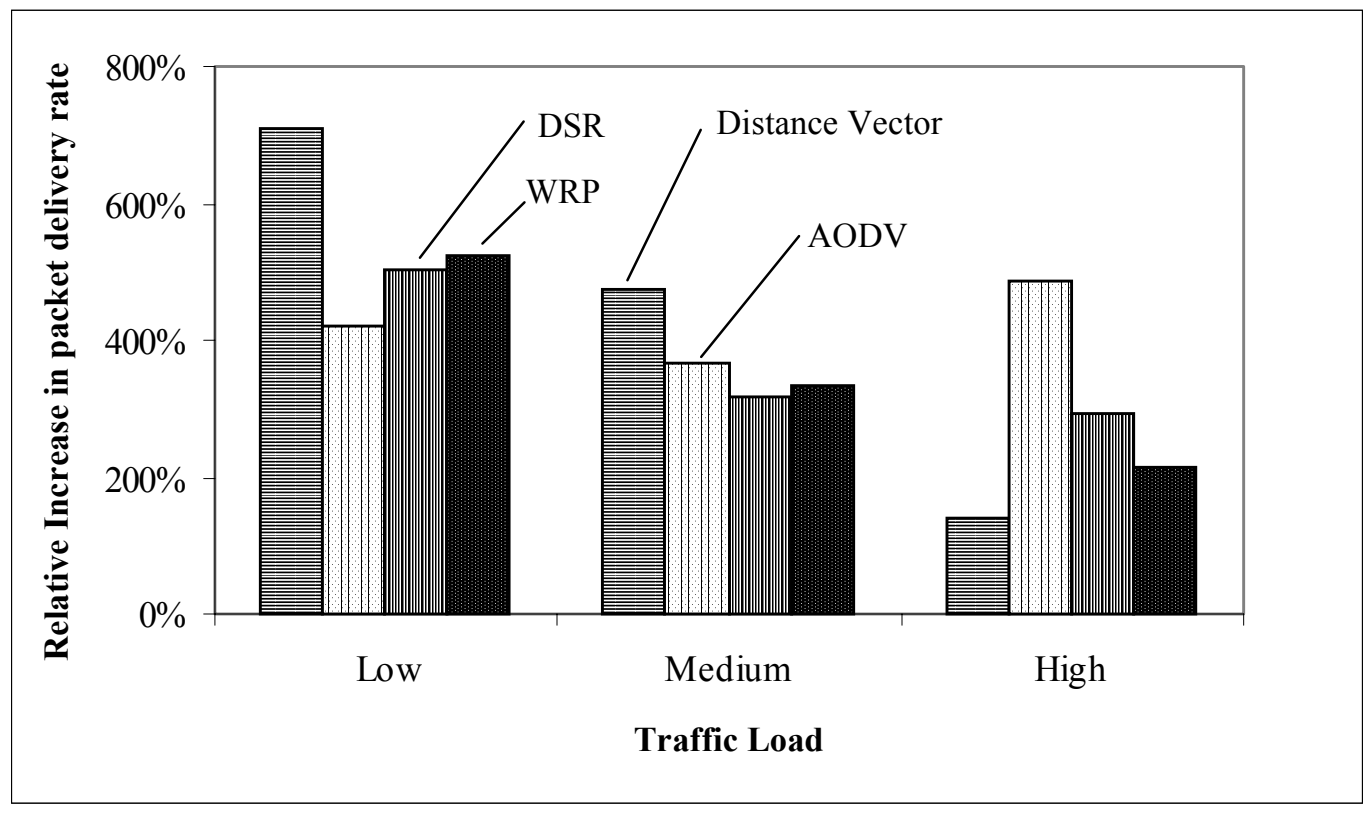

Figure 4 - The relative increase in packet delivery rate when mobility was decreased to zero mobility 
Author Name: Fujinoki, Hiroshi

E-mail: hfujino@siue.edu

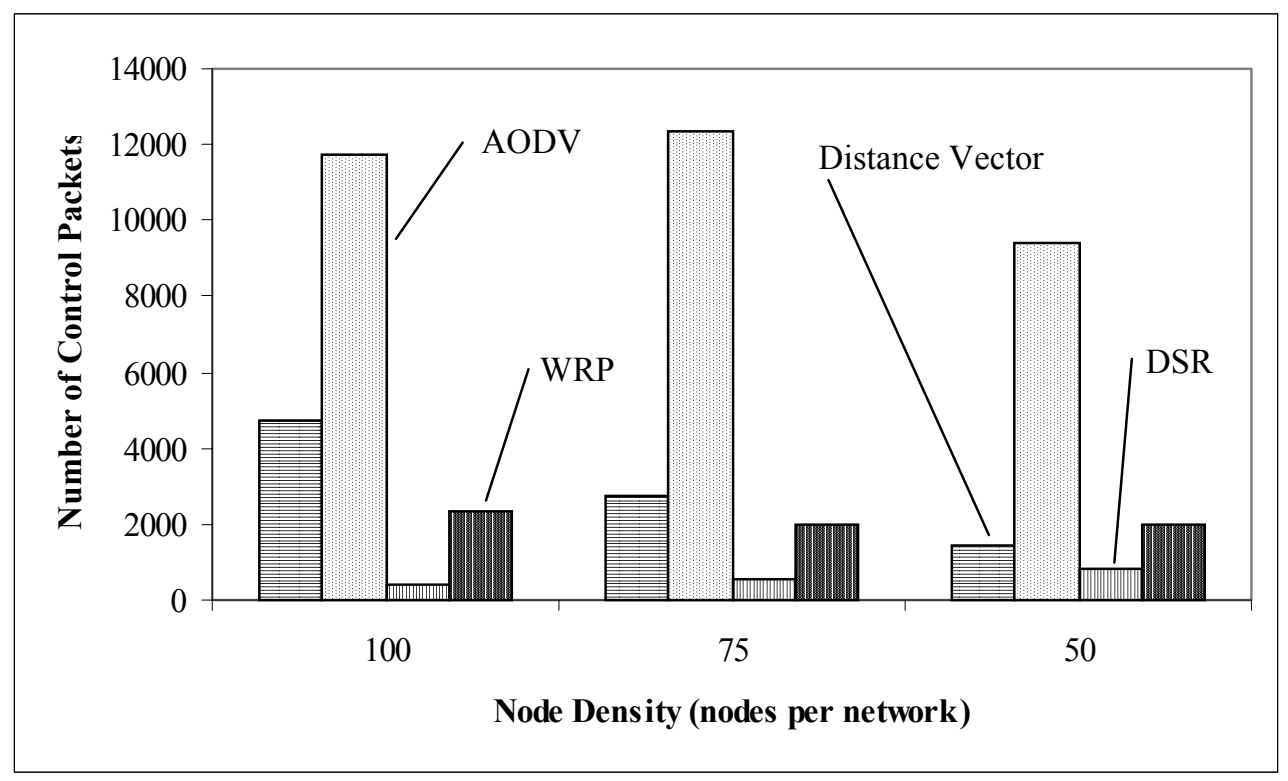

Figure 5 - Messaging overhead for three different levels of node density at high traffic load 
Author Name: Fujinoki, Hiroshi

E-mail: hfujino@siue.edu

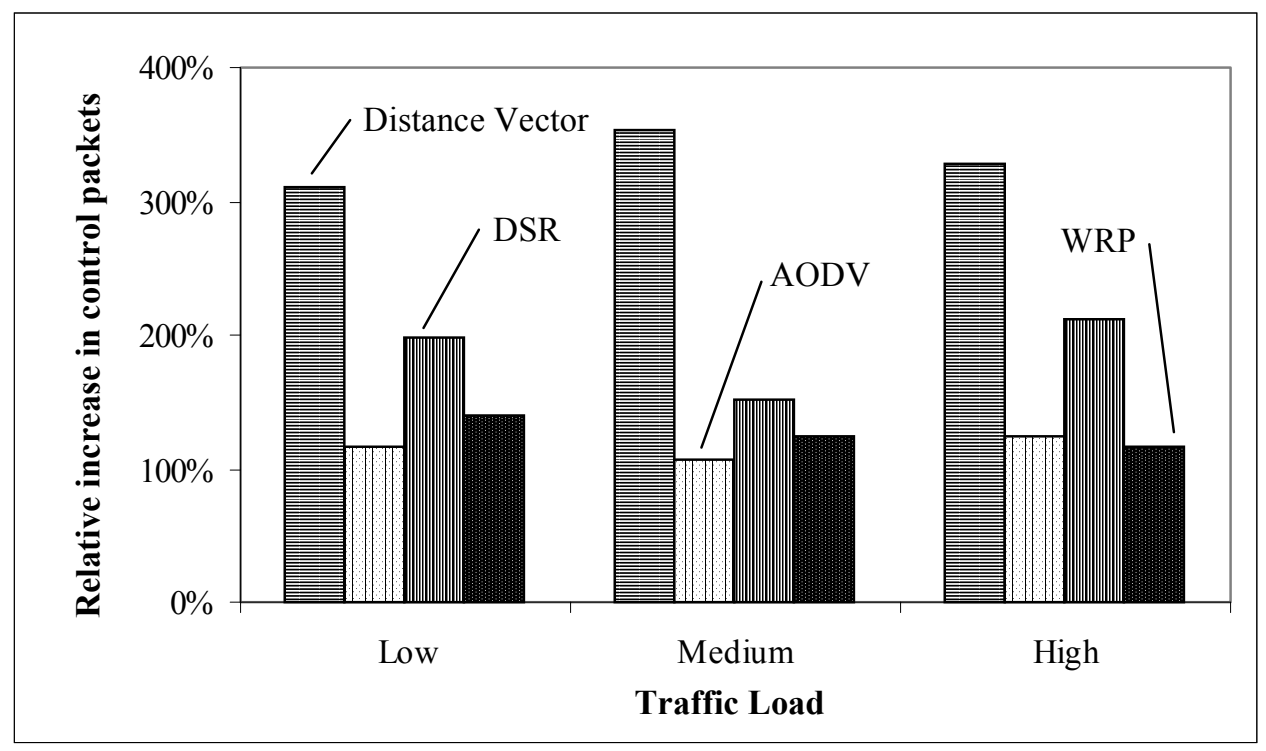

Figure 6 - The ratio of the number of control packets observed at high node density to that observed at low node density 
Author Name: Fujinoki, Hiroshi

E-mail: hfujino@siue.edu

\begin{tabular}{|l|l|l|l|l|}
\hline \multicolumn{1}{|c|}{ Properties } & \multicolumn{1}{c|}{ DV } & \multicolumn{1}{c|}{ DSR } & \multicolumn{1}{c|}{ AODV } \\
\hline Type of routing & Proactive & Proactive & Reactive & Reactive \\
\hline Distributed & YES (hop-by-hop) & YES (hop-by-hop) & NO (source routing) & YES (hop-by-hop) \\
\hline Routing loops & Possible & Not Possible & Not Possible & Not Possible \\
\hline Use of broadcast & No & No & Yes & Yes \\
\hline $\begin{array}{l}\text { Control } \\
\text { Overhead }\end{array}$ & $\begin{array}{l}\text { Constant to the } \\
\text { number of sessions }\end{array}$ & $\begin{array}{l}\text { Constant to the } \\
\text { number of sessions }\end{array}$ & $\begin{array}{l}\text { Affected by the } \\
\text { number of sessions }\end{array}$ & $\begin{array}{l}\text { Affected by the } \\
\text { number of sessions }\end{array}$ \\
\hline Routing entries & All destinations & All destinations & Destinations in use & Destinations in use \\
\hline Alternative paths & Not available & Not available & Available & Available \\
\hline Request response & Short & Short & Long (if not cached) & Long (if not cached) \\
\hline Advantages & $\begin{array}{l}\text { Short response time } \\
\text { Low message OH }\end{array}$ & Short response time & Quick path recovery & $\begin{array}{l}\text { Small routing table } \\
\text { Quick recovery }\end{array}$ \\
\hline Disadvantages & $\begin{array}{l}\text { Routing loops } \\
\text { Large routing table } \\
\text { Long convergence } \\
\text { time }\end{array}$ & Large routing table & $\begin{array}{l}\text { Long response time } \\
\text { Large packet header }\end{array}$ & $\begin{array}{l}\text { Long response time } \\
\text { Aggregate routing is } \\
\text { not possible at } \\
\text { intermediate nodes }\end{array}$ \\
\hline
\end{tabular}

Table 1 - Major properties of the four protocols compared 
Author Name: Fujinoki, Hiroshi

E-mail: hfujino@siue.edu

\begin{tabular}{|l|l|r|r|r|r|r|r|r|r|}
\hline & & \multicolumn{9}{|c|}{ Node Mobility } & \multicolumn{3}{c|}{ Node Density } \\
\hline Load & Protocols & Perpetual & \multicolumn{1}{|c|}{ High } & \multicolumn{1}{|c|}{ Medium } & \multicolumn{1}{c|}{ Low } & \multicolumn{1}{c|}{ Zero } & \multicolumn{1}{c|}{ High } & \multicolumn{1}{c|}{ Medium } & Low \\
\hline Low & DV & 1,577 & 1,121 & 967 & 750 & 624 & 4,456 & 2,598 & 1,438 \\
\hline & WRP & 3,159 & 2,037 & 2,013 & 1,687 & 1,276 & 2,512 & 2,073 & 1,800 \\
\hline & AODV & 281 & 157 & 156 & 157 & 156 & 307 & 303 & 262 \\
\hline & DSR & 108 & 145 & 132 & 69 & 52 & 95 & 46 & 48 \\
\hline & & & & & & & & & \\
\hline Medium & DV & 1,552 & 1,086 & 947 & 766 & 647 & 4,434 & 2,600 & 1,254 \\
\hline & WRP & 3,112 & 2,020 & 1,730 & 1,404 & 1,285 & 2,432 & 2,197 & 1,967 \\
\hline & AODV & 1,805 & 751 & 627 & 482 & 365 & 1,241 & 1,201 & 1,156 \\
\hline & DSR & 452 & 357 & 408 & 383 & 351 & 284 & 318 & 187 \\
\hline & & & & & & & & & \\
\hline High & DV & 1,568 & 1,166 & 979 & 927 & 799 & 4,742 & 2,723 & 1,444 \\
\hline & WRP & 2,875 & 1,943 & 1,749 & 1,757 & 1,246 & 2,300 & 1,995 & 1,980 \\
\hline & AODV & 10,123 & 5,097 & 4,901 & 4,797 & 3,899 & 11,768 & 12,333 & 9,431 \\
\hline & DSR & 822 & 741 & 821 & 789 & 522 & 828 & 543 & 393 \\
\hline
\end{tabular}

Table 2 - Number of control packets observed for the five different levels of node mobility and three different levels of node density 
Author Name: Fujinoki, Hiroshi

E-mail: hfujino@siue.edu

\begin{tabular}{|c|l|c|c|c|c|c|c|c|c|}
\hline & & \multicolumn{9}{|c|}{ Node Mobility } & \multicolumn{3}{c|}{ Node Density } \\
\hline Load & Protocols & Perpetual & High & Medium & Low & Zero & High & Medium & Low \\
\hline Low & DV & $12.3 \%$ & $16.4 \%$ & $34.6 \%$ & $47.4 \%$ & $86.9 \%$ & $24.2 \%$ & $16.8 \%$ & $16.3 \%$ \\
\hline & WRP & 17.1 & 18.4 & 38.7 & 64.9 & 89.1 & 26.3 & 22.2 & 22.4 \\
\hline & AODV & 13.8 & 16.3 & 30.3 & 38.4 & 57.9 & 21.8 & 17.1 & 19.1 \\
\hline & DSR & 12.0 & 20.1 & 28.8 & 41.2 & 60.3 & 20.0 & 14.6 & 16.2 \\
\hline & & & & & & & & & \\
\hline Medium & DV & $14.6 \%$ & $16.6 \%$ & $29.5 \%$ & $44.2 \%$ & $69.4 \%$ & $23.2 \%$ & $15.0 \%$ & $11.3 \%$ \\
\hline & WRP & 23.0 & 36.6 & 52.7 & 55.0 & 76.6 & 27.1 & 18.2 & 21.0 \\
\hline & AODV & 19.6 & 32.9 & 38.5 & 45.2 & 71.6 & 26.0 & 21.1 & 13.3 \\
\hline & DSR & 19.5 & 21.8 & 28.8 & 35.3 & 62.2 & 25.0 & 18.3 & 12.7 \\
\hline & & & & & & & & & \\
\hline High & DV & $10.4 \%$ & $11.2 \%$ & $14.0 \%$ & $12.7 \%$ & $14.4 \%$ & $13.8 \%$ & $11.3 \%$ & $11.4 \%$ \\
\hline & WRP & 8.9 & 13.4 & 15.8 & 18.0 & 19.1 & 19.9 & 13.3 & 13.0 \\
\hline & AODV & 5.4 & 12.9 & 16.8 & 19.8 & 26.0 & 9.3 & 8.3 & 8.1 \\
\hline & DSR & 6.2 & 9.6 & 13.4 & 12.5 & 18.1 & 15.5 & 8.0 & 10.2 \\
\hline
\end{tabular}

Table 3 - Packet delivery rate (in percent) for the five different levels of node mobility and three levels of node density 
Author Name: Fujinoki, Hiroshi

E-mail: hfujino@siue.edu

\begin{tabular}{|c|l|c|c|c|c|c|c|c|c|}
\hline & & \multicolumn{9}{|c|}{ Node Mobility } & \multicolumn{3}{c|}{ Node Density } \\
\hline Load & Protocols & Perpetual & High & Medium & Low & Zero & High & Medium & Low \\
\hline Low & DV & 0.28 & 0.28 & 0.15 & 0.13 & 0.12 & 0.12 & 0.19 & 0.17 \\
\hline & WRP & 0.15 & 0.13 & 0.10 & 0.10 & 0.10 & 0.11 & 0.15 & 0.13 \\
\hline & AODV & 0.56 & 0.47 & 0.53 & 0.46 & 0.43 & 0.40 & 0.36 & 0.41 \\
\hline & DSR & 2.79 & 2.89 & 2.10 & 1.44 & 1.52 & 1.72 & 1.54 & 1.40 \\
\hline & & & & & & & & & \\
\hline Medium & DV & 0.28 & 0.25 & 0.19 & 0.14 & 0.12 & 0.12 & 0.18 & 0.14 \\
\hline & WRP & 0.21 & 0.17 & 0.15 & 0.12 & 0.11 & 0.11 & 0.19 & 0.14 \\
\hline & AODV & 0.26 & 0.20 & 0.19 & 0.20 & 0.20 & 0.25 & 0.26 & 0.22 \\
\hline & DSR & 1.16 & 1.82 & 1.15 & 1.03 & 1.10 & 0.28 & 0.49 & 0.44 \\
\hline & & & & & & & & & \\
\hline High & DV & 32.42 & 5.02 & 4.54 & 4.72 & 4.58 & 2.58 & 4.60 & 5.17 \\
\hline & WRP & 3.83 & 2.59 & 2.47 & 2.31 & 2.56 & 3.12 & 5.59 & 3.40 \\
\hline & AODV & 18.04 & 5.34 & 5.05 & 5.09 & 6.77 & 8.08 & 8.64 & 5.88 \\
\hline & DSR & 15.50 & 7.75 & 7.12 & 6.03 & 4.65 & 7.02 & 10.83 & 7.48 \\
\hline
\end{tabular}

Table 4 - Mean end-to-end delay (in seconds) for the five different levels of node mobility and node density 